emeraldine salt) mixed with carbon black, the team obtained experimental thresholds of 10 ppt (parts per trillion) for butylamine and 1 ppt for cadaverine, while the thresholds for the non-amine vapors were comparable to those of the previous device built. For comparison, the human detection threshold for butylamine has been reported to be between 0.1 and $1 \mathrm{ppm}$.

Iulia C. Muntele

\section{Strong Directional Emission Observed in Lasing Characteristics of a Pendant Drop Deformed by an Applied Electric Field}

In an article published in the April 1 issue of Optics Letters, Xiao-Yun Fu and Wing-Kee Lee from the Chinese University of Hong-Kong, Department of Physics, report the results of their studies of the lasing properties of an oval-shaped resonant cavity (ORC) with a continuously variable aspect ratio. The ORC was formed with a dye-doped pendant drop (rhodamine 6G dye in an ethylene glycol solution) placed inside a variable static electric field. Pumped with a nitrogen laser, the ORC drop was found to have strong directional emission characteristics and an intensity enhancement factor as great as 19.5, results that are in good agreement with the theoretical calculations. The anisotropy was found to increase as the eccentricity was increased. Also, the lasing threshold was found to decrease with the increase in eccentricity; for $e=0$, the threshold was $6.2 \mu \mathrm{J}$, while for $e=0.38$, the threshold dropped to $3.8 \mu \mathrm{J}$. Although no previous investigation on the optical properties of a continuously deformable pendant drop has been reported, tiny whispering-gallery-type lasers, such as microdisk, microcylinder, and microdroplet (circulatory symmetric resonators) have been previously studied, and are known to have two intrinsic disadvantages: the lack of high output power and directional emission. The use of asymmetric resonant cavities is believed to overcome these disadvantages.

Claudiu Muntele

\section{Uniform Monolayer of Organic Molecules Provides Precise Control of Semiconductor Device Properties}

The functions of organic molecules are so diverse that their inclusion in electronics would provide an extensive range of possibilities. However, the observation of these molecules' electrical properties has been impeded by incongruities in the structure of organic molecules themselves. Layers of organic molecules that are used in this kind of research contain "pinholes" which are small defects that are very difficult to detect but radically sway conductance. Determining whether measurements resulted from the passage of the current through the organic molecules or through a pinhole was very difficult.

Researchers at the Weizmann Institute have found that changing the type of organic molecules in a monolayer led to a predictable, systematic change in the electrical characteristics of a semiconductor device. Not only were the researchers able to control the properties of the semiconductor in the device, but they also were able to predict the kind of control that would be exerted by different types of organic molecules over the device.

The researchers chose to analyze the molecules indirectly by focusing on the influence that the molecules were suspected to have on semiconductors. Using a series of molecules synthesized by Abraham Shanzer of the Weizmann Institute's Organic Chemistry Department, Ayelet Vilan, a graduate student working with David Cahen of the Materials and Interfaces Department, constructed a onemolecule-thick layer of very short organic molecules. The organic materials used were the so-called "di-carboxylic acids," which are tartaric acid derivatives; tartaric acid is also known as "fruit acid," and found, for example, in wine. Vilan placed the monolayer on a common semiconductor, GaAs, and directed an electric current through it. The monolayer was so thin that, for the most part, the electric current passed by the molecules without interacting with them. In an article published in the March 9 issue of Nature, the researchers report that this fact meant that it was of minimal importance if the electrons went via a molecule or a pinhole. According to the researchers, though, it is important to note that while the organic molecules barely affect the passage of the electrical current through them, they very much influence the electron affinity of the semiconductor, the energy that an electron gains when it comes from infinity into the semiconductor's conduction band.

Along with the decision to work with monolayers of organic molecules, Vilan developed a method for preparing semiconductor devices. The technique is founded on a widely used semiconductor device (diode), which is comprised of a semiconductor connected to a metal. She inserted the di-carboxylic acid organic monolayer between these two components-placed by self-assembly of the molecules on the GaAs. Since the organic molecules were sandwiched between the semiconductor and the metal sheet, it was critically important to ensure that the delicate monolayer would not be crushed underneath the metal sheet. Vilan used a thin gold leaf as the metal sheet and gently floated it onto the monolayer. Thus, the monolayer remained intact. Vilan said that this study "provides new insights into the emerging field of molecular electronics. So little is known about the effects that occur between molecules and the electric conductors we normally use. This approach may provide a basis for the design of novel types of semiconductorbased devices, from improvements in relatively simple devices such as solar cells, to possible new types of computer chips."

\section{$\mathrm{Sn} / \mathrm{Li}_{2} \mathrm{O}$ Nanocomposites Synthesized as Negative Electrode Materials for Lithium-Ion Batteries}

A team of researchers, D.L. Foster, J. Wolfenstine, J.R. Read, and W.K. Behl, at the U.S. Army Research Laboratory, reported a method for the preparation of lithium oxide/tin nanocomposites in the May issue of Electrochemical and Solid-State Letters which show promising properties as electrode materials. The straightforward synthesis yields tin particles on the order of $100 \mathrm{~nm}$ or less which are uniformly distributed within a lithium oxide matrix. The composites showed improved performance as electrode materials compared to other $\mathrm{SnO}$ electrodes with the elimination of most of the irreversible capacity on the first cycle.

In an effort to enhance safety and rechargeability of lithium batteries, pure lithium anodes have been replaced by other lithium-containing compounds in recent years. Among the promising candidates are lithium/tin oxide nanocomposites that are transformed to tin/ lithium oxide composites during the first cycle. This reduction "leads to a large irreversible capacity loss on the first discharge cycle," said Foster. Therefore, the team investigated the possibility of using a chemical reaction to form a tin/lithium oxide nanocomposite.

The successful process utilizes the commercially available precursors lithium nitride and tin oxide. The reactants are jar milled under a dry argon atmosphere for five days at room temperature, leading to the formation of tin, lithium oxide, and nitrogen gas. X-ray diffraction confirmed that the product contained a mixture of crystalline tin and an amorphous component that is most likely lithium oxide. 
Scanning electron microscopy (SEM) imaging showed that most of the tin particles fall in the size range below $100 \mathrm{~nm}$ with fairly round shapes and a uniform distribution throughout the $\mathrm{Li}_{2} \mathrm{O}$ matrix. "But apparently, there are still enough large tin particles to account for the rather narrow x-ray intensity peaks," said Wolfenstine. Electrical measurements resulted in curves for the jar milled composite and a $\mathrm{SnO}$ electrode that are very similar, but as expected the milled sample shows only little irreversible capacity loss. This indicates that the milling of $\mathrm{Li}_{3} \mathrm{~N}$ and $\mathrm{SnO}$ is a viable process for the formation of $\mathrm{Sn} / \mathrm{Li}_{2} \mathrm{O}$ nanocomposites that avoids the difficult milling of soft and ductile tin itself.

The researchers agree that there is still room for optimization "such as controlling the temperature during the milling process, adding an inert material such as $\mathrm{Al}_{2} \mathrm{O}_{3}$ or $\mathrm{Li}_{2} \mathrm{O}$ to the starting materials before milling, or starting with a tin-oxide glass or tin-oxide composite glass" in an effort to achieve even smaller particle sizes.

CORA LIND

\section{Rod-Shaped CdSe \\ Nanocrystals Produced}

To date, experimental nanocrystals fashioned from semiconductors have been shaped like dots or spheres. However, researchers at Lawrence Berkeley National Laboratory and the University of California-Berkeley have produced CdSe nanocrystals that are shaped like rods as reported in the March 2 issue of Nature.

"We have demonstrated that controlling the kinetics of semiconductor nanocrystal growth can be used to vary the shapes of the resulting particles from a nearly spherical morphology to a rod-like one," said Paul Alivisatos, the leader of the experimental team who holds a joint appointment with Berkeley Lab's Materials Sciences Division, and with the UC Berkeley Chemistry Department. "These rod-like semiconductor nanocrystals may prove advantageous in biological labeling experiments and as chromophores in light-emitting diodes."

By carefully maintaining a relatively fast rate of growth in the right mix of surfactant, the researchers could induce crystals of a selected size to assume an elongated rod-like faceted shape that maximized crystal surface area. Subsequent tests showed that these rod-shaped nanocrystals emit light that is polarized along their long-axis in contrast to the nonpolarized light fluoresced by cadmium-selenide nanocrystal dots.

"Polarized emission along the long axis of these rods should be in biological tag- ging experiments where the orientation of the tag needs to be determined," said Alivisatos.

Other tests showed that the gap between emission and absorption energies is larger for nanocrystal rods than for nanocrystal dots, which Alivisatos said should be an advantage in applications such as light-emitting diodes (LEDs) where the re-absorption of light can be a problem. It was also shown that the multiple rods could be packed and aligned, another advantage for both LEDs and for the use of these rods in photovoltaic cells.

\section{Interactions of Synthesized Magmas Indicate the Importance of Chemical Reactions in the Formation of Volcanic Edifices}

Recent experiments by Craig Lundstrom of the University of Illinois have shed light on how glassy materials are formed in exotic chunks of mantle called xenoliths, and how ascending magmas in the mantle can affect the lava output at Earth's surface through chemical, rather than thermal, reactions.

Lundstrom, a professor of geology, said, "Sodium in ascending magma can quickly diffuse into the surrounding mantle at lower pressures, fundamentally altering the process by which the mantle melts. Sodium infiltration can account for the creation of silica-rich glasses in xenoliths, and for the anomalous mineralogical composition of mantle found beneath mid-ocean ridges."

Xenoliths are pieces of mantle that get pulled off and are carried to the surface during a volcanic eruption. Within the xenoliths are former melts-now found as glasses-which differ radically from the magma that typically emanates from the mantle, basalt. The glasses reside between the two primary silicate minerals of the uppermost mantle, olivine and orthopyroxene.

"Xenoliths have been found in alkalirich magmas from ocean island and continental volcanic settings worldwide," Lundstrom said. "But the origin of the glassy regions, and why they have peculiar elemental compositions, have been poorly understood."

As reported in the February 3 issue of Nature, to study the potential chemical interaction, Lundstrom first synthesized samples of magma found deep in the

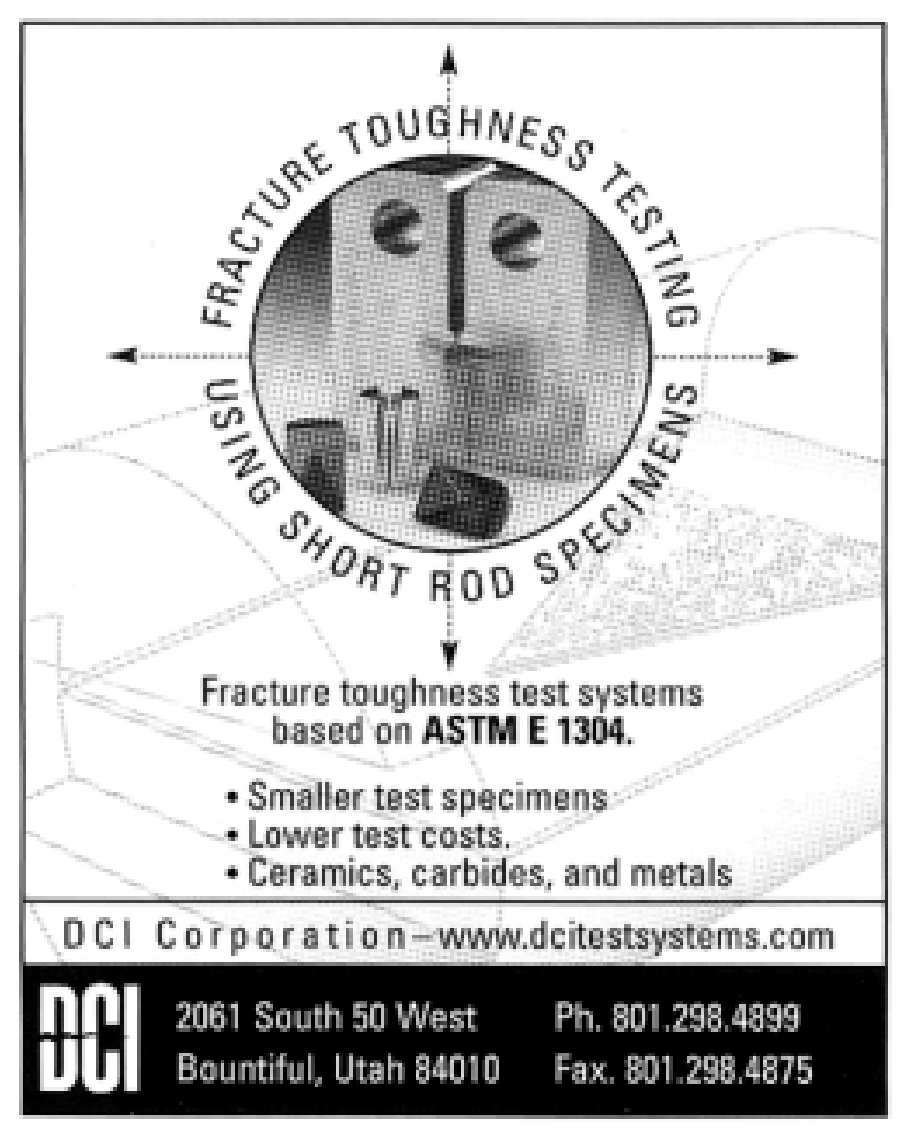

Circle No. 10 on Inside Back Cover 